(C) Masson, Paris, 1980.

Annales de Parasitologie (Paris)

1980 , t. $55, \mathrm{n}^{\circ} 4$, pp. $427-443$.

\title{
Sur les Nématodes Echinonematinae
}

\author{
par A.-G. CHABAUD (1), Cl. SEUREAU (2), I. BEVERIDGE (3), \\ O. BAIN (1) et M.-Cl. DURETTE-DESSET (1)
}

(1) Laboratoire de Zoologie-Vers, associé au C.N.R.S., Muséum National d'Histoire Naturelle, 43, rue Cuvier, F 75231 Paris Cedex 05.

(2) Laboratoire d'Histophysiologie fondamentale et appliquée, ERA 570 C.N.R.S., Université $P$. et $M$. Curie, Paris.

(3) Institute of Medical and Veterinary Sc., Adelaide, S.A.

RESUME. Des données complémentaires sur les Echinonematinae ont été obtenues par l'étude de la Collection Helminthologique Australienne et par l'étude morphologique et biologique d'un nouveau matériel.

Les Echinonematinae actuellement connus sont les suivants :

- Seurechina chaneeti n. gen., n. sp., parasite de Dasyurus hallucatus (West Australia), n'a pas de gros crochets céphaliques, mais environ 50 rangées d'épines couvrant le $1 / 5$ antérieur du corps.

- Inglechina australis (Inglis et Mawson, 1967) n. gen., n. comb., parasite de Sminthopsis crassicaudata (South Australia), a 3 rangs de gros crochets céphaliques, mais n'a pas de spécialisation de la région cervicale.

- Echinonema v. Linstow, 1898, est parasite de Dasyuridae et de Peramelidae. La tête porte 3 rangs de gros crochets et la région cervicale environ 15 rangs de crochets d'assez grande taille. Les femelles sont monodelphes.

- E. edmondsi n. sp., parasite de Dasyurus hallucatus (Northern Territory) a un œsophage long et des épines céphaliques plus longues sur le $1^{\mathrm{er}}$ rang que sur le $2^{\mathrm{e}}$.

- E. cinctum von Linstow, 1898, parasite d'Isoodon macrourus dans le Nord-Est de l'Australie, a un œsophage long et des épines céphaliques plus longues sur le $2^{\mathrm{e}}$ rang que sur le $1^{\text {er }}$.

- E. meridionalis n. sp., parasite d'Isoodon obesulus dans le Sud-Est de l'Australie, a un œsophage court et des épines ventrales précloacales.

Accepté le 5 février 1980. 
- E. inglisi n. sp. (= E. cinctum sensu Inglis, 1967), parasite d'Isoodon obesulus dans l'Ouest de l'Australie, a un œsophage court et des épines somatiques limitées aux 2/3 antérieurs du corps chez le mâle.

- E. spp. : les extrémités postérieures mâles d'Echinonema spp., parasites d'Isoodon en Tasmanie et de Perameles spp. sur le continent sont figurées. Il s'agit vraisemblablement d'autres espèces.

- E. cinctum évolue expérimentalement chez l'Orthoptère Locusta migratoria. Les réactions cellulaires (simple capsule hémocytaire) et la morphologie du stade infestant sont primitives, et paraissent intermédiaires entre le type Subuluroïde et le type Seuratoïde.

Les Echinonematinae sont interprétés comme issus d'ancêtres proches des Seuratum (parasites de Chiroptères), qui se seraient adaptés à un groupe particulier de Marsupiaux australiens, en perfectionnant leur appareil de fixation, mais leurs caractéristiques biologiques restent très primitives.

\section{Studies on the Echinonematinae (Nematoda).}

SUMMARY. Complementary data on the Echinonematinae have been obtained by study of "The Australian Helminth Collection, formerly the University of Adelaide, Department of Zoology Helminth Collection, currently housed in the South Australian Museum ", and by a morphological and biological study of new material.

The Echinonematinae actually known are the following:

- Seurechina chaneeti n. gen., n. sp., parasite of Dasyurus hallucatus (West Australia) does not have large cephalic hooks but about 50 rings of spines covering the anterior fifth of the body.

- Inglechina australis (Inglis and Mawson, 1967) n. gen., n. comb., a parasite of Sminthopsis crassicaudata (South Australia) has 3 rings of large cephalic hooks, but no special cuticular structures in the cervical region.

- Echinonema v. Linstow, 1898, is a parasite of Dasyuridae and Peramelidae. The head bears 3 rings of large hooks and the cervical region about 15 rings of hooks of medium size. The females are monodelphic.

- E. edmondsi $n$. sp. from Dasyurus hallucatus (Northern Territory) has a long oesophagus and cephalic hooks of the first circle longer than those of the second one.

- E. cinctum von Linstow, 1898, from Isoodon macrourus (N.E. of Australia) has a long oesophagus and cephalic hooks of the second circle longer than those of the first one.

- E. meridionalis n. sp., from Isoodon obesulus (S.E. of Australia) has a short oesophagus and precloacal spines in males.

- E. inglisi $n$. sp. (= E. cinctum sensu Inglis, 1967), from Isoodon obesulus (Western Australia) has a short oesophagus and no precloacal spines in males. 
- E. spp.: the different shapes of the tail in males are illustrated for E. sp. from Perameles and for E. sp. from Isoodon of Tasmania.

- E. cinctum developed experimentally in the Orthoptera Locusta migratoria. The cellular reactions (simple haemocytic capsule) and the morphology of the infective stage are primitive and seem to be intermediate between the Subuluroid and Seura. toid types.

The Echinonematinae are interpreted as having been derived from ancestors close to Seuratum (parasite of bats), which adapted to a particular group of Australian marsupials and evolved a characteristic structure for attachment to host tissues. However they have retained a primitive biology.

Le genre Echinonema Linstow, 1898, placé selon les auteurs dans différentes familles de Spiruroidea, a été reconnu comme Seuratoidea (Inglis, 1967), et a été classé dans la famille des Seuratidae (Quentin, 1971).

La récolte d'un nouveau matériel, l'étude de l'évolution larvaire d'une espèce chez un Orthoptère, et la révision des spécimens de la Collection Helminthologique Australienne (1), permettent de compléter nos connaissances sur ces Nématodes particulièrement intéressants par leurs caractères archaïques.

\section{I. - Seurechina chaneeti n. g., n. sp. (fig. 1)}

\section{Matériel :}

3 et $1 \delta^{\star}$ parasites de Dasyurus hallucatus Gould, 1842, récoltés par G. de Chaneet à Koolan Is, off Derby (West Australia). Holo et allotype déposés au South Australian Museum (Adelaïde).

\section{Description :}

- Femelle holotype: à l'exception de la région céphalique, le corps est entièrement couvert d'épines, alignées transversalement sur chaque interstrie. Sur une longueur de $750 \mu \mathrm{m}$, la portion antérieure porte environ 50 rangs de grosses épines coalescentes (fig. $1 \mathrm{~F}$ ); en arrière de cette zone, les épines sont beaucoup plus petites (fig. $1 \mathrm{G}$ ), à l'exception de la lèvre antérieure de la vulve où se trouvent à nouveau quelques grosses épines. En avant du corps, il y a 42 épines sur chaque rang tranversal, environ 2 fois plus au niveau de la fin de l'œsophage, puis à nouveau environ 40 au niveau des petites épines. Tête libre d'épines sur une hauteur de $50 \mu \mathrm{m}$, portant un plateau céphalique ovalaire allongé dans le sens latéral $(65 \mu \mathrm{m} \times 45 \mu \mathrm{m})$. Seules les 4 papilles céphaliques et les amphides sont visibles. Extrémité antérieure

(1) Primitivement Collection Helminthologique du Département de Zoologie de l'Université d'Adélaïde, actuellement hébergée dans le South Australian Museum. 
de l'œsophage surmontée d'une cupule chitinoïde élargie d'arrière en avant, surtout dans le sens dorso-ventral. Les parois de cette cupile sont constituées par deux anneaux superposés, un postérieur haut de $5 \mu \mathrm{m}$, un antérieur haut de $10 \mu \mathrm{m}$. Diamètres internes de la cupule de $5 \mu \mathrm{m} \times 25 \mu \mathrm{m}$ en arrière, et de $10 \mu \mathrm{m} \times 50 \mu \mathrm{m}$ en avant. Sous l'hypoderme céphalique existente 4 paires sublatérales de lames hautes de $90 \mu \mathrm{m}$ (fig. $1 \mathrm{~B}, C$ et $E$ ), qui paraissent être des dilatations des champs latéraux et jouent peut-être un rôle dans le mécanisme de fixation des crochets cervicaux. Esophage simple, claviforme. Vulve post-équatoriale s'ouvrant en arrière d'une grosse lèvre saillante. Didelphie.
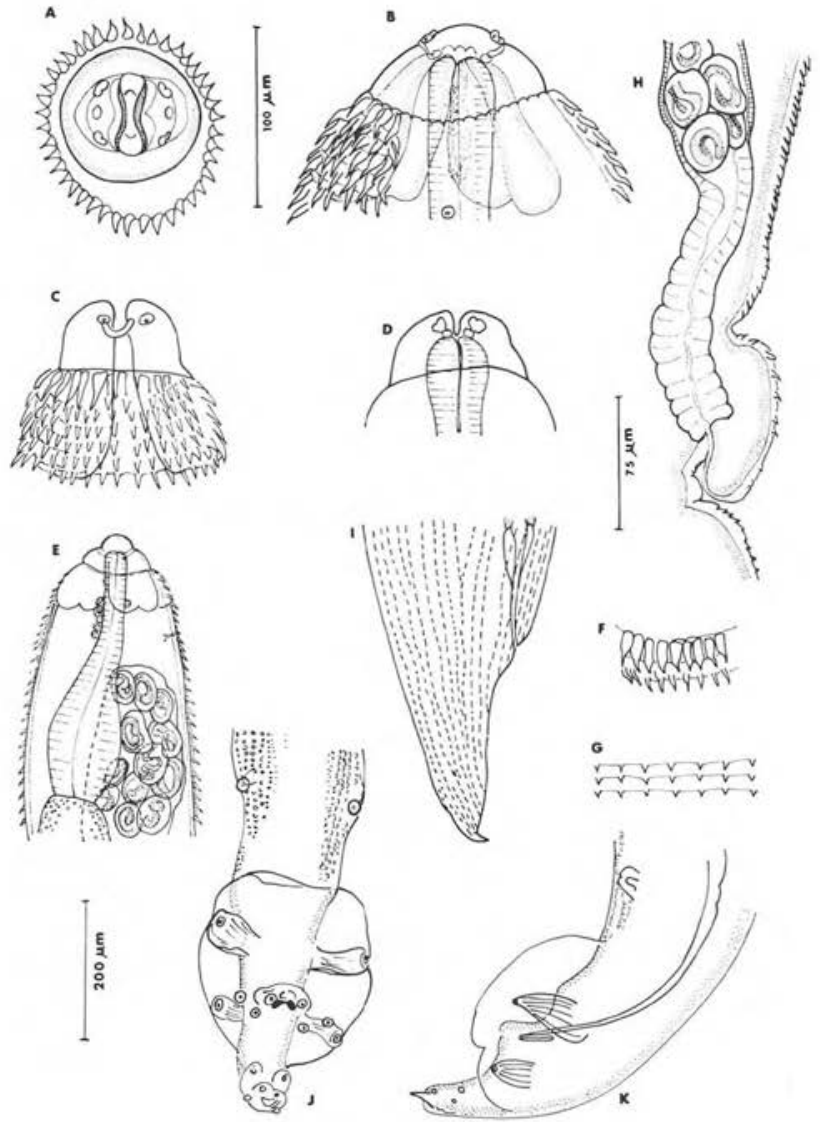

Fig. 1. Seurechina chaneeti

A : $\uparrow$, tête, vue apicale; B : id., vue latérale; C : id., vue médiane ; D : id., coupe optique ; $\mathrm{E}: \mathcal{\wp}$, extrémité antérieure, vue latérale; $\mathrm{F}: \mathfrak{+}$, épines de la région antérieure du corps;

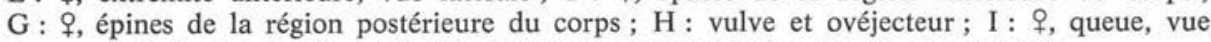
latérale; $\mathrm{J}: \delta^{\widehat{t}}$, extrémité postérieure, vue ventrale ; $\mathrm{K}: i d$., vue latérale.

A, B, C, D, F, G, I, J, K : échelle $100 \mu \mathrm{m}$; E : échelle $200 \mu \mathrm{m}$; H : échelle $75 \mu \mathrm{m}$. 
Dimensions: longueur du corps $3,8 \mathrm{~mm}$; largeur $220 \mu \mathrm{m}$; anneau nerveux, deirides et pore excréteur respectivement à 95, 110 et $150 \mu \mathrm{m}$ de l'apex. Esophage long de $350 \mu \mathrm{m}$. Vulve à $2,25 \mathrm{~mm}$, ovaire antérieur à $1,90 \mathrm{~mm}$ et ovaire postérieur à 2,95 mm de l'extrémité antérieure. Eufs : $40 \times 35 \mu \mathrm{m}$. Queue longue de $120 \mu \mathrm{m}$.

- Mâle allotype: longueur 3,1 mm, largeur $170 \mu \mathrm{m}$. Anneau nerveux et deirides respectivement à $70 \mu \mathrm{m}$ et $80 \mu \mathrm{m}$ de l'apex. Esophage long de $280 \mu \mathrm{m}$. Spicules longs de $185 \mu \mathrm{m}$. Gubernaculum faible, long de $20 \mu \mathrm{m}$. Spinulation somatique se terminant par 2 bandes latérales de petites formations arrondies respectant les faces médianes. Extrémité postérieure figurée en $1 \mathrm{~J}$ et $1 \mathrm{~K}$. La lèvre supérieure du cloaque est enflée sur ses bords latéraux, simulant ainsi une paire de papilles supplémentaires, alors qu'en réalité, il n'y a que 4 paires péri-cloacales comme chez Echinonema. La $2^{\mathrm{e}}$ paire précloacale, très largement dilatée, soutient les ailes caudales.

\section{Discussion :}

L'espèce est proche de Seuratum ou d'Echinonema, mais la structure buccale, la spinulation et l'extrémité postérieure mâle sont originales.

Nous devons créer pour elle un nouveau genre, Seurechina (dédié à L.-G. Seurat). Définition: Seuratidae, Echinonematinae. Corps couvert de rangées d'épines plus grandes sur le cinquième antérieur du corps. Capsule buccale bien développée, allongée dans l'axe médian. Vulve post-équatoriale. Didelphie. Ailes caudales du đo bien développées.

Espèce type : Seurechina chaneeti n. gen., n. sp., parasite de Dasyuridae.

\section{II. - Inglecbina australis}

(Inglis et Mawson, 1967) n. gen., n. comb.

(= Echinonema australis Inglis et Mawson, 1967)

L'espèce est décrite de Sminthopsis crassicaudata (Gould, 1844) d'Oodnadatta (South Australia) et est très remarquable par l'absence de crochets différenciés dans la région cervicale.

Les auteurs ont placé l'espèce dans le genre Echinonema, mais, pour rendre compte de l'évolution du groupe et pour mieux opposer les espèces à appareil de fixation incomplet aux espèces à appareil de fixation complet, à la fois céphalique et cervical, il nous semble intéressant de créer, pour cette espèce, un genre particulier, Inglechina $\mathrm{n}$. gen.

Définition: Seuratidae, Echinonematinae. Tête armée de 3 rangs de crochets céphaliques. Région cervicale non dilatée, avec spinulation simple du même type que celle du corps. Bouche triangulaire. Vulve pré-équatoriale.

Espèce type: Inglechina australis (Inglis et Mawson, 1967), n. comb., parasite de Dasyuridae. 


\section{III. - Genre Ecbinonema Linstow, 1898}

\section{A. Caractères généraux.}

Les nombreux spécimens examinés sont proches les uns des autres.

Depuis le travail original de von Linstow, aucune espèce n'a été décrite en détail. Il est donc utile de préciser quelques éléments morphologiques concernant la spinulation et l'appareil génital femelle.

1. Spinulation: trois rangs de très grands crochets sur le bulbe céphalique, cinq à six rangs de très petits sur le cou, deux rangs de taille intermédiaire, puis environ onze rangs de grands crochets sur une dilatation cuticulaire intéressant la région œsophagienne. Viennent ensuite deux rangs de crochets de taille intermédiaire, puis un rang de très petits crochets sur chaque interstrie tout le long du corps.

Tous les rangs de l'extrémité antérieure, ainsi que les 50 premiers rangs de petits crochets somatiques, comptent chacun environ 14 crochets chez le $\delta$ et 16 chez la ๆ. Plus postérieurement, de nouvelles files de crochets naissent à mi-distance entre les files primaires. Le nombre de crochets par rang s'accroît donc progressivement et atteint, à la partie moyenne du corps, environ 46 éléments chez le mâle, 54 chez la femelle, puis diminue vers l'extrémité postérieure. Les files d'épines se poursuivent jusqu'à la pointe caudale chez la femelle. Chez le mâle, au contraire, les épines dorsales s'effacent à partir du quart postérieur du Ver. Cette atrophie des épines gagne progressivement la face ventrale, mais il reste cependant quelques files d'épines ventrales qui s'hypertrophient légèrement et qui ne disparaissent qu'au niveau de la première paire de papilles cloacales.

2. Appareil génital femelle: la vulve s'ouvre un peu en avant du milieu du corps. L'ovéjecteur est dirigé vers l'avant; il n'y a qu'un seul utérus. Tous les œufs mûrs sont donc dans la région antérieure du corps ; la fin de l'utérus, l'oviducte et l'ovaire sont localisés dans la région postérieure.

\section{B. Caractères particuliers.}

Des différences morphologiques légères, mais assez constantes d'un spécimen à l'autre, s'observent selon la nature de l'hôte et selon la répartition géographique.

a. Les parasites d'Isoodon macrourus du Nord-Est de l'Australie (N.T., Q.L.D., Nord du N.S.W.), ont pour caractère le plus évident un œsophage long se terminant au niveau des derniers rangs des épines cervicales.

b. Les parasites d'Isoodon obesulus du Sud-Est de l'Australie (Sud du N.S.W., S.A., Vic.), ont pour caractère le plus évident un œsophage court, se terminant au niveau des grandes épines cervicales.

Il importe de déterminer à laquelle de ces deux formes doit être attribué le taxon de Linstow. La provenance de l'hôte «Perameles obesula Geoffroy » n'est pas 
indiquée directement dans le texte, mais le matériel provient de l'expédition du Docteur Richard Semon et les mammifères correspondants ont été étudiés par Römer (1894). Le texte de celui-ci indique que "Perameles obesula " a été récolté à Burnett, c'est-à-dire à environ $300 \mathrm{~km}$ au Nord de Brisbane. Dans la nomenclature actuelle, l'hôte paraît donc correspondre à Isoodon macrourus (Gouldt, 1842) (voir Mackerras et Mackerras, 1960).

La publication originale est difficile à interpréter, car la figure $\mathrm{XXXV}, 3$, montre un œsophage court, mais le texte indique un œsophage long (1,8 mm dans les 2 sexes). Il ne reste donc que le lieu de récolte pour tenter d'identifier l'espèce type.

Cependant le taxon est repris en 1926 par Yorke et Maplestone, avec une nouvelle description précise. Johnston et Mawson (1939) indiquent que le matériel de Yorke et Maplestone provient de Townsville (Q.L.D.). L'œsophage est long.

Nous préférons donc utiliser le taxon d'E. cinctum pour les spécimens à œsophage long provenant du Nord-Est de l'Australie.

L'analyse des documents bibliographiques et l'examen morphologique des collections conduit à individualiser les formes suivantes :

1. (4) Esophage long se terminant au niveau des épines cervicales les plus postérieures.

2. (3) Epines céphaliques du $1^{\mathrm{er}}$ rang plus longues que celles du $2^{\mathrm{e}}$ rang. Paire de papilles latérales précloacales très développées. 5 à 6 rangs d'épines ventrales précloacales terminales. Parasite de Dasyurus hallucatus, Northern Territory, E. edmondsi n. sp.

3. (2) Epines céphaliques du $1^{\mathrm{er}}$ rang plus courtes que celles du $2^{\mathrm{e}}$ rang. Paire de papilles latérales précloacales pas beaucoup plus grandes que les autres paires. 3-4 rangs d'épines ventrales précloacales terminales. Parasite d'Isoodon macrourus dans le Nord-Est de l'Australie. E. cinctum Linstow, 1898.

4. (1) EEsophage court se terminant au niveau des épines cervicales moyennes.

5. (6) Mâle avec épines ventrales préclocales présentes. Parasite d'Isoodon obesulus dans le Sud-Est de l'Australie. E. meridionalis n. sp.

6. (5) Mâle avec épines somatiques limitées aux 2/3 antérieurs du corps. Parasite d'Isoodon obesulus en Australie occidentale. E. inglisi n. sp. $(=$ E. cinctum sensu Inglis, 1967).

\section{Morphologie des espèces.}

\section{Echinonema edmondsi n. sp. (fig. 2).}

Matériel type: $\sigma^{t}$ holotype, $q$ allotype: South Australian Museum. Paratypes: M.N.H.N. Paris.

Estomac et intestin grêle de Dasyurus hallucatus Gould, 1842, capturé à Cannon Hill (Northern Territory), le 20-X-1972. 


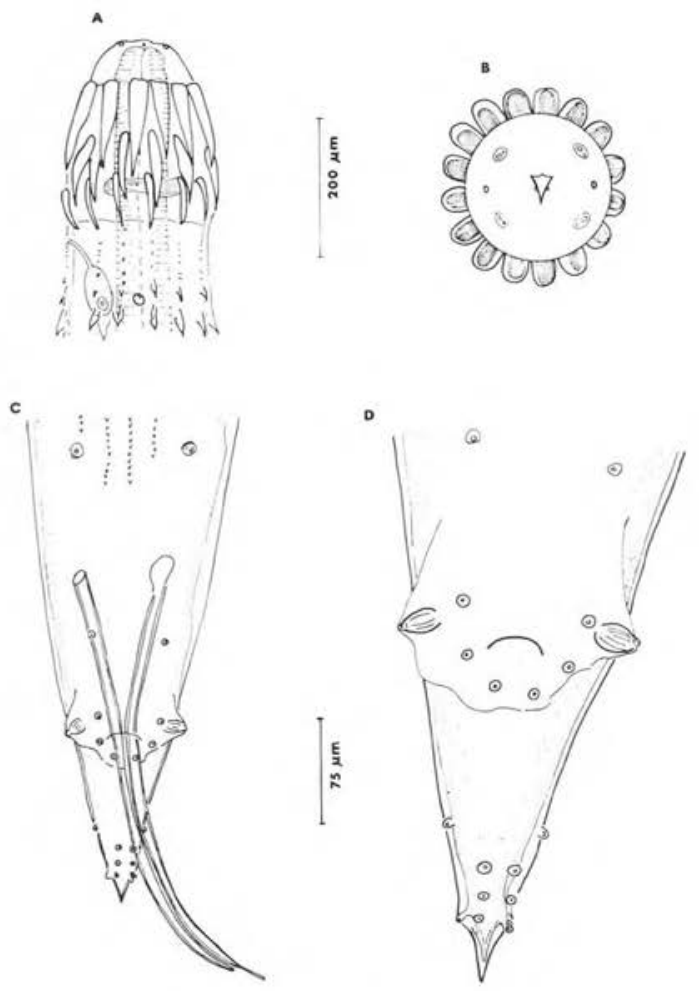

Fig. 2. Echinonema edmondsi

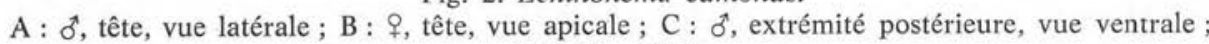
D : id., détail.
A, B, C : échelle $200 \mu \mathrm{m} ; \mathrm{D}$ : échelle $75 \mu \mathrm{m}$.

Autre matériel : $\delta$ et $q$, même hôte et même localisation à Nourlangie (Northern Territory), 21-VIII-1979.

Description: bouche triangulaire avec bord dorsal plus court que les bords latéroventraux.

\section{- Mâle holotype:}

Corps long de 11,8 mm, large de $350 \mu \mathrm{m}$. Esophage $1300 \mu \mathrm{m}$. Anneau nerveux, pore excréteur et deirides respectivement à $220 \mu \mathrm{m}, 280 \mu \mathrm{m}$ et $370 \mu \mathrm{m}$ de l'apex. Début et fin du bulbe céphalique à 60 et $200 \mu \mathrm{m}$ de l'extrémité antérieure. Crochets céphaliques du $1^{\mathrm{er}}, 2^{\mathrm{e}}$ et $3^{\mathrm{e}}$ rang, longs respectivement de $135 \mu \mathrm{m}, 110 \mu \mathrm{m}$ et $75 \mu \mathrm{m}$. Gubernaculum de $78 \mu \mathrm{m}$. Queue longue de $230 \mu \mathrm{m}$. Epines ventrales se terminant sur 4-5 files au niveau de la $1^{\text {re }}$ paire de papilles cloacales. Disposition des papilles cloacales figurée en $2 C, D$. La paire de papilles latérales précloacales, très développées, soulève 2 petites ailes cuticulaires. 
- Femelle allotype :

Corps long de $22 \mathrm{~mm}$, large de $500 \mu \mathrm{m}$. Esophage long de $1450 \mu \mathrm{m}$. Anneau nerveux, pore excréteur et deirides respectivement à $290 \mu \mathrm{m}, 320 \mu \mathrm{m}$ et $360 \mu \mathrm{m}$ de l'apex. Début et fin du bulbe céphalique à $80 \mu \mathrm{m}$ et $275 \mu \mathrm{m}$ de l'extrémité antérieure. Crochets céphaliques du $1^{\mathrm{er}}, 2^{\mathrm{e}}$ et $3^{\mathrm{e}}$ rang, longs respectivement de 170,142 et $95 \mu \mathrm{m}$. Vulve à $9 \mathrm{~mm}$ de l'extrémité antérieure. Monodelphie. Eufs sphériques ou un peu ovoïdes, de $48 \mu \mathrm{m}$ de diamètre. Queue longue de $640 \mu \mathrm{m}$.

2. Echinonema cinctum (Linstow, 1898) (nec Inglis, 1967) (fig. 3).

$$
\text { (= Hoplocephalus cinctus Linstow, 1898) }
$$

Matériel examiné : nombreux spécimens parasites d'Isoodon macrourus (Gould, 1842), de différentes localités :

N.T. : Woolwonga, Darwin.

Q.L.D. : Innisfail, Townsville, Ingham, Brisbane.

Nord du N.S.W. : Lismore.
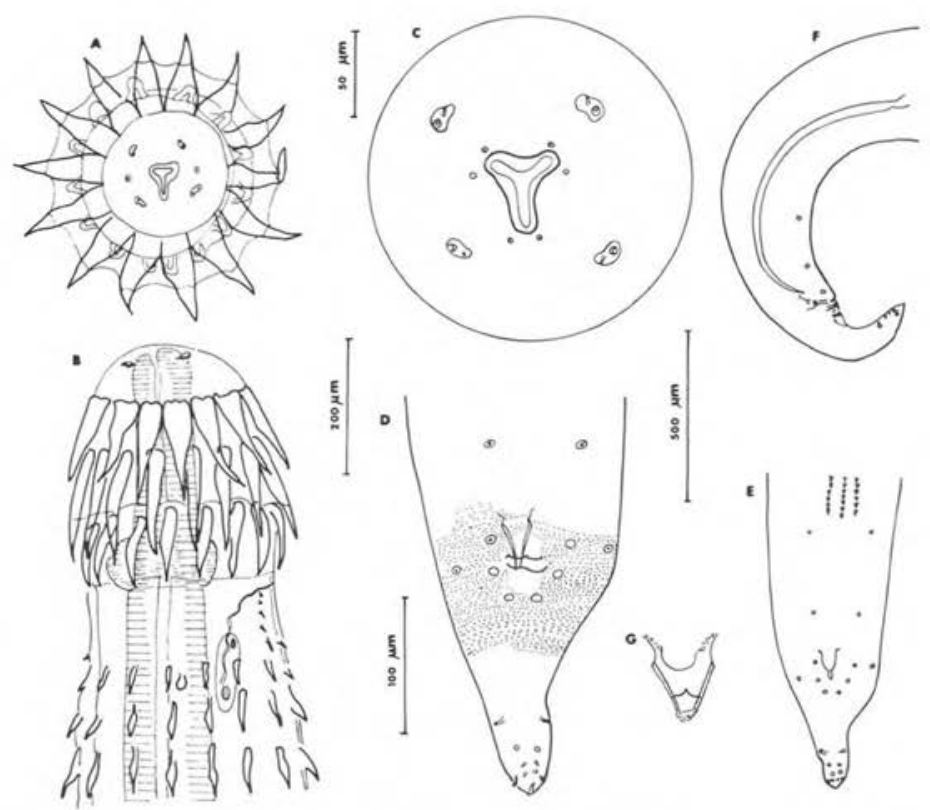

Fig. 3. Echinonema cinctum $\sigma^{*}$

A : tête, vue apicale; B : extrémité antérieure, vue latérale; C: détail région céphalique, vue apicale; D : extrémité postérieure, vue ventrale; E : id., vue d'ensemble; F : id., vue latérale; G: Gubernaculum, vue ventrale.

A, B, D : échelle $200 \mu \mathrm{m} ; \mathrm{C}$ : échelle $50 \mu \mathrm{m} ; \mathrm{E}, \mathrm{F}$ : échelle $500 \mu \mathrm{m}$; G : échelle $100 \mu \mathrm{m}$. 
Redescription: (spécimens 49 HD de Townsville).

- Mâle:

Corps long de $16 \mathrm{~mm}$, large de $430 \mu \mathrm{m}$. Bulbe céphalique débutant à $84 \mu \mathrm{m}$, se terminant à $340 \mu \mathrm{m}$ de l'apex. Crochets du $1^{\text {er }}, 2^{\mathrm{e}}$ et $3^{\mathrm{e}}$ rang, longs respectivement de $140 \mu \mathrm{m}, 170 \mu \mathrm{m}$ et $105 \mu \mathrm{m}$. Anneau nerveux, pore excréteur et deirides à $310 \mu \mathrm{m}$, $360 \mu \mathrm{m}$ et $490 \mu \mathrm{m}$ de l'extrémité antérieure. Esophage de $1325 \mu \mathrm{m}$. Extrémité postérieure et disposition des papilles cloacales figurées en $3 D, E, F$. Queue longue de $340 \mu \mathrm{m}$. Spicules et gubernaculum respectivement longs de $920 \mu \mathrm{m}$ et $62 \mu \mathrm{m}$.

\section{- Femelle :}

Corps long de $34 \mathrm{~mm}$, large de $850 \mu \mathrm{m}$. Début et fin du bulbe céphalique à 100 et $440 \mu \mathrm{m}$ de l'apex. Anneau nerveux, pore excréteur et deirides respectivement à 360,400 et $580 \mu \mathrm{m}$ de l'extrémité antérieure.

Crochets du $1^{\mathrm{er}}, 2^{\mathrm{e}}$ et $3^{\mathrm{e}}$ rang, longs respectivement de $180 \mu \mathrm{m}, 210 \mu \mathrm{m}, 150 \mu \mathrm{m}$. Zone cuticulaire dilatée de la région œsophagienne débutant à $600 \mu \mathrm{m}$ et finissant à $2200 \mu \mathrm{m}$ de l'apex. Esophage long de $2000 \mu \mathrm{m}$. Vulve s'ouvrant à $15 \mathrm{~mm}$ de l'extrémité antérieure. Eufs arrondis de $42 \mu \mathrm{m}$ de diamètre. Queue à pointe aiguë, longue de $600 \mu \mathrm{m}$.

3. Echinonema meridionalis n. sp. (fig. 4).

\section{Matériel :}

Cotypes: nombreux spécimens parasites d'Isoodon obesulus (Shaw et Nodder, 1797), de Waitpinga, S.A. (HC 4460). Dépôt South Australian Museum et M.N.H.N., $\mathrm{n}^{\circ} 236$ H.D.

Autre matériel: nombreux spécimens du même hôte, provenant de Myponga S.A., H.C. 4446.

Description :

- Mâle :

Corps long de $9,25 \mathrm{~mm}$, large de $350 \mu \mathrm{m}$. Crochets du $1^{\text {er }}, 2^{\mathrm{e}}$ et $3^{\mathrm{e}}$ rang, longs respectivement de 160, 180 et $100 \mu \mathrm{m}$. Esophage de $950 \mu \mathrm{m}$. Anneau nerveux, pore excréteur et deirides respectivement à 220 , 350 et $410 \mu \mathrm{m}$ de l'apex. Queue longue de $200 \mu \mathrm{m}$. Spicules et gubernaculum respectivement longs de 830 et $60 \mu \mathrm{m}$.

\section{- Femelle:}

Corps long de $27 \mathrm{~mm}$, large de $600 \mu \mathrm{m}$. Crochets du $1^{\text {er }}, 2^{\mathrm{e}}$ et $3^{\mathrm{e}}$ rang, longs respectivement de 170, 200 et $130 \mu \mathrm{m}$. Anneau nerveux, pore excréteur et deirides respectivement à 290,340 et $500 \mu \mathrm{m}$ de l'apex. Esophage long de $1100 \mu \mathrm{m}$. Vulve s'ouvrant à 10,8 mm de l'extrémité antérieure. CEufs ovales de $45 \times 32 \mu \mathrm{m}$. Queue longue de $950 \mu \mathrm{m}$. L'espèce semble également monodelphe, mais nous n'avons pas de matériel dont la fixation permette une dissection. 


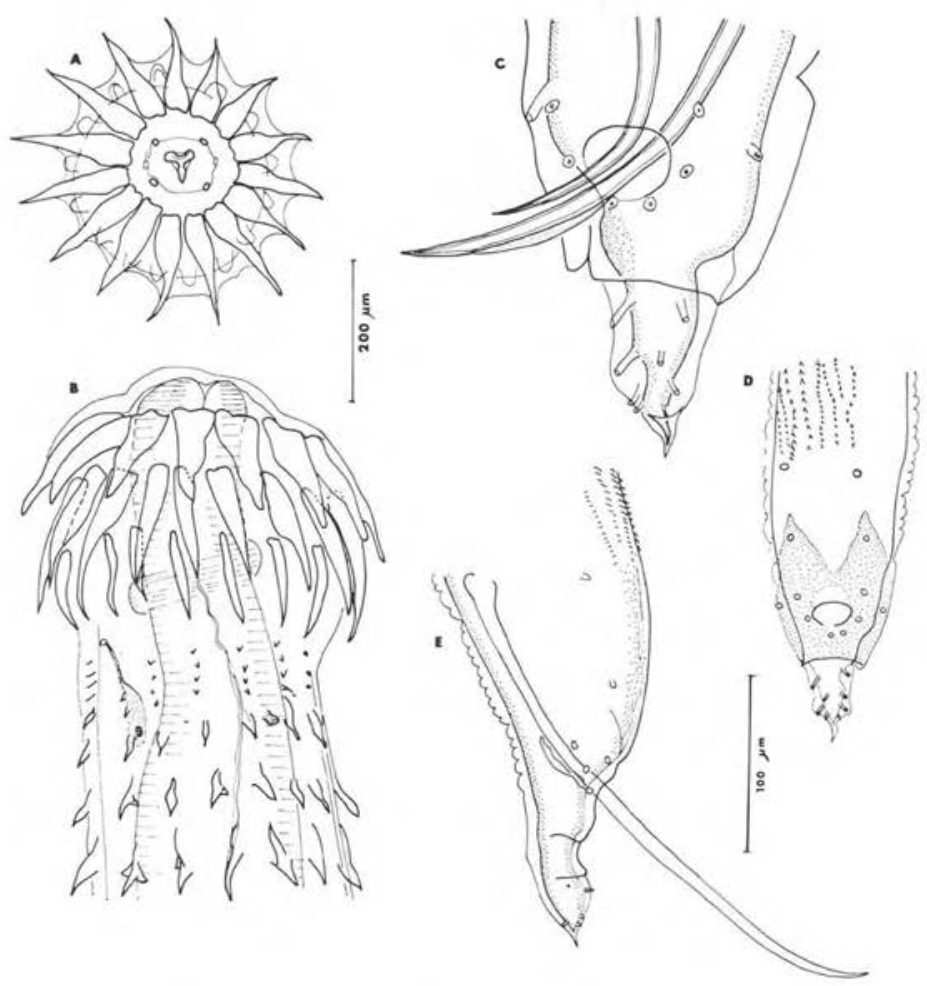

Fig. 4. Echinonema meridionalis

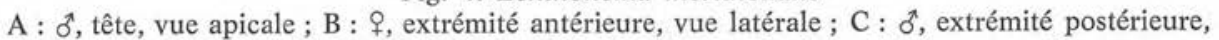
vue ventrale; D: id., vue d'ensemble; $\mathrm{E}: i d$., vue latérale d'un autre spécimen.

A, B, D, E : échelle $200 \mu \mathrm{m} ; \mathrm{C}$ : échelle $100 \mu \mathrm{m}$.

\section{Echinonema inglisi n. sp.}

$$
\text { (=E. cinctum sensu Inglis, 1967) }
$$

Inglis (1967), en s'intéressant surtout à la structure céphalique, décrit brièvement sous le nom d'E. cinctum des spécimens provenant d'Isoodon obesulus de Perth (Australie Occidentale).

L'espèce évoque un peu $E$. edmondsi par le grand développement de la paire de papilles latérales précloacales, mais l'œsophage est court, les épines céphaliques du $1^{\text {er }}$ rang plus courtes que celles du $2^{\mathrm{e}}$ rang, et, surtout, les épines somatiques du mâle s'arrêtent aux $2 / 5$ du corps et ne se prolongent pas sur la ligne médio-ventrale. Nous en concluons qu'il s'agit d'une espèce distincte, que nous nommons: Echinonema inglisi $\mathrm{n}$. sp. 
5. Echinonema spp. (fig. 5).

Il est très vraisemblable que d'autres espèces restent à décrire.

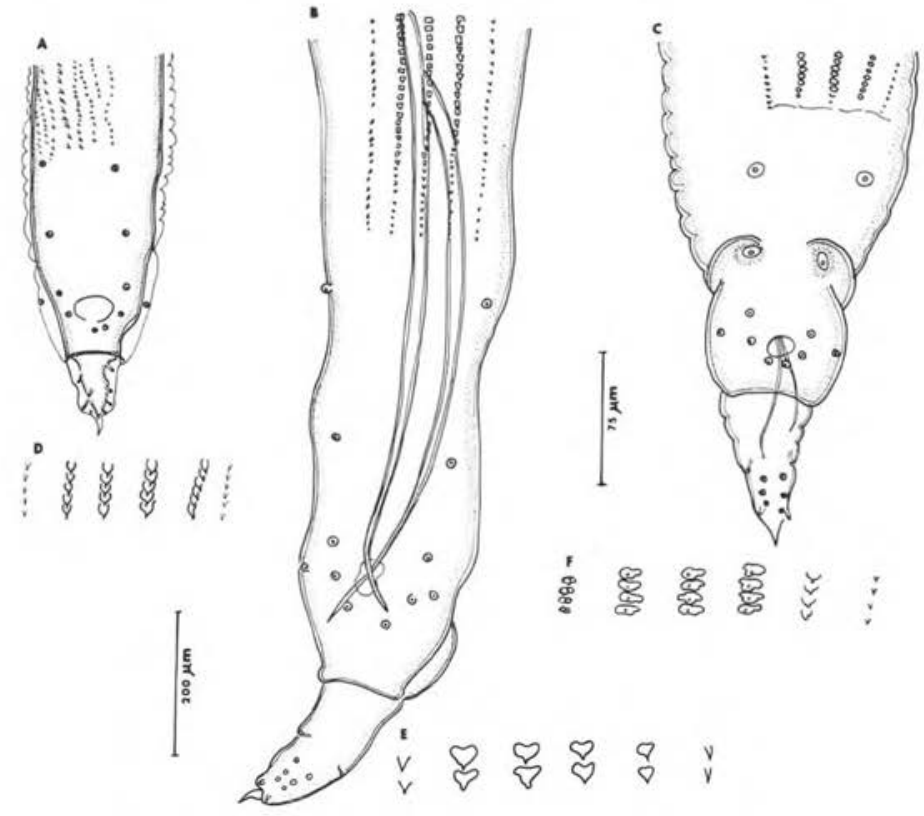

Fig. 5. Variations de l'extrémité postérieure des $\delta^{\star}$ d'Echinonema

A, B, C : extrémités postérieures des $\delta$, vues ventrales; échelle : $200 \mu \mathrm{m}$. D.E.F. : détail des derniers rangs d'épines ventrales; échelle: $75 \mu \mathrm{m}$.
A, D : E. meridionalis. Isoodon, S.A.
B, E : E. sp. 1. Isoodon, Tasmanie.
C, F : E. sp. 2. Perameles nasuta.

Les nématodes étant plus ou moins contractés, l'orientation des épines et les distances qui les séparent varient légèrement d'un spécimen à l'autre.

Nous figurerons, à la même échelle, les extrémités postérieures des mâles, et les derniers rangs d'épines ventrales pour:

- E. meridionalis, parasite d'Isoodon obesulus (H.C. 4460), à Waitpinga, S.A. (fig. 5 A et $D$ ).

- E. sp., parasite du même hôte (H.C. 4530), à Upper Dromedary, Tas. (fig. 5 B et $E$ ).

- E. sp., parasite de Perameles nasuta Geoffroy, 1804 (H.C. 1820), localité inconnue (fig. $5 \mathrm{C}$ et $F$ ). (D'autres spécimens du même hôte et qui paraissent avoir des caractères comparables ont été décrits par Johnston et Mawson, 1940). 
Les différences paraissent importantes entre ces différents lots, mais nous ne disposons pas d'un matériel suffisant pour en faire des descriptions détaillées.

De la même façon, le spécimen récolté chez Dasyurus viverrinus (Shaw, 1800) dans la région de Sydney, qui a été signalé par Johnston et Mawson, 1939, est une femelle juvénile qui ne peut être déterminée.

\section{IV. - Développement larvaire d'Echinonema cinctum}

Cinq femelles mûres sont prélevées à l'autopsie d'un Isoodon macrourus de Townsville, le 12-11-78 et conservées dans de l'eau ordinaire. Le 4-12-78, 10 Locusta migratoria d'élevage sont infestées par voie buccale avec des fragments d'utérus contenant des œufs mûrs. Le 12-12-78, une Locusta sacrifiée montre de très nombreuses larves (environ 100), du début du $2^{\mathrm{c}}$ stade, encapsulées contre la paroi de la partie postérieure du mésentéron. L’intestin postérieur, en arrière des tubes de Malpighi, est négatif. Le 21-12-78 (17 $\mathrm{e}$ jour), les Locusta contiennent de nombreuses larves infestantes encapsulées au niveau du mésentéron.

Un Marsupial américain, Metachirops opossum, est infesté par voie buccale avec de nombreuses larves infestantes; il est négatif à l'autopsie, le 28-5-79.

\section{Morphologie (fig. 6).}

\section{- Larve du début du $2^{\mathrm{c}}$ stade :}

Une larve de ce stade est figurée en $6 A, D$. Un spécimen, long de $980 \mu \mathrm{m}$, est large de $80 \mu \mathrm{m}$. Esophage long de $130 \mu \mathrm{m}$. Capsule buccale : $3 \mu \mathrm{m}$. Anneau nerveux et pore excréteur respectivement à $68 \mu \mathrm{m}, 110 \mu \mathrm{m}$ de l'extrémité antérieure. Queue $105 \mu \mathrm{m}$, ébauche génitale à $350 \mu \mathrm{m}$ de la pointe caudale.

\section{- Larve du $3^{\mathrm{e}}$ stade :}

Une larve de ce stade est figurée en 6 E, $K$. Un spécimen long de $900 \mu \mathrm{m}$ est large de $95 \mu \mathrm{m}$. Esophage long de $160 \mu \mathrm{m}$. Capsule buccale $3 \mu \mathrm{m}$. Anneau nerveux et pore excréteur respectivement à 60 et $105 \mu \mathrm{m}$ de l'extrémité antérieure. Queue $105 \mu \mathrm{m}$; ébauche génitale à $325 \mu \mathrm{m}$ de la pointe caudale. La partie antérieure et interne de l'œsophage est revêtue sur chaque lobe d'une petite lame chitinoïde, haute de 4 à $5 \mu \mathrm{m}$. La pointe caudale est armée de quelques minuscules épines.

\section{- Discussion :}

L'élément atypique dans ce développement larvaire est qu'il n'y a pas de croissance en longueur, mais seulement en largeur durant le second stade larvaire. Ceci évoque un Subulure, plutôt qu'un Seuratoïde. De même, l'habitus de la larve infestante subglobuleuse, avec tête et queue fortement courbées vers la face dorsale, donne une image intermédiaire entre celle d'un Subulura (voir Quentin, 1969, fig. 3 A) et celle d'un Seuratum (voir Quentin et Seureau, 1975, fig. 3 F). 

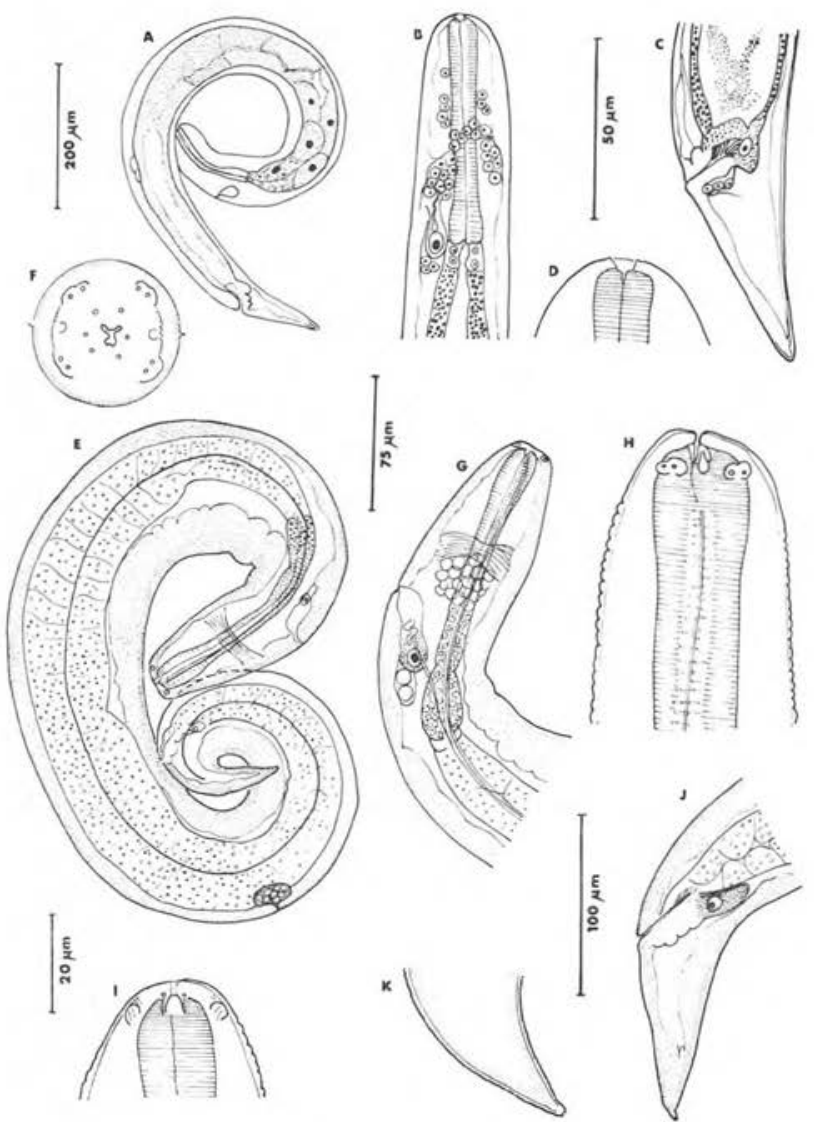

Fig. 6. Echinonema cinctum

A-D : larve du début du $2^{\mathrm{e}}$ stade ( $8^{e}$ jour après l'infestation). A : vue latérale ; B : extrémité antérieure, vue latérale; C: extrémité postérieure, vue latérale; D: tête, vue latérale. E-K : larve infestante $\left(17^{\circ}\right.$ jour après l'infestation); E : vue latérale : F : tête, vue apicale ; $\mathrm{G}$ : extrémité antérieure, vue latérale; $\mathrm{H}$ : région céphalique, vue latérale; $\mathrm{I}$ : id., vue dorsale ; J : extrémité postérieure, vue latérale ; $\mathrm{K}$ : pointe caudale.

A : échelle $200 \mu \mathrm{m} ; \mathrm{B}, \mathrm{C}, \mathrm{G}, \mathrm{J}$ : échelle $100 \mu \mathrm{m}$; D : échelle $50 \mu \mathrm{m}$; E : échelle $75 \mu \mathrm{m}$; F, H, I, K : échelle $20 \mu \mathrm{m}$.

Réactions cellulaires de l'hôte ( $f i g .7$ ).

Les parasites encapsulés sont accolés à la paroi de l'intestin moyen du Criquet, du côté de l'hémocœle, et, pour la plupart, juste en avant des ampoules collectrices des tubes de Malpighi. 

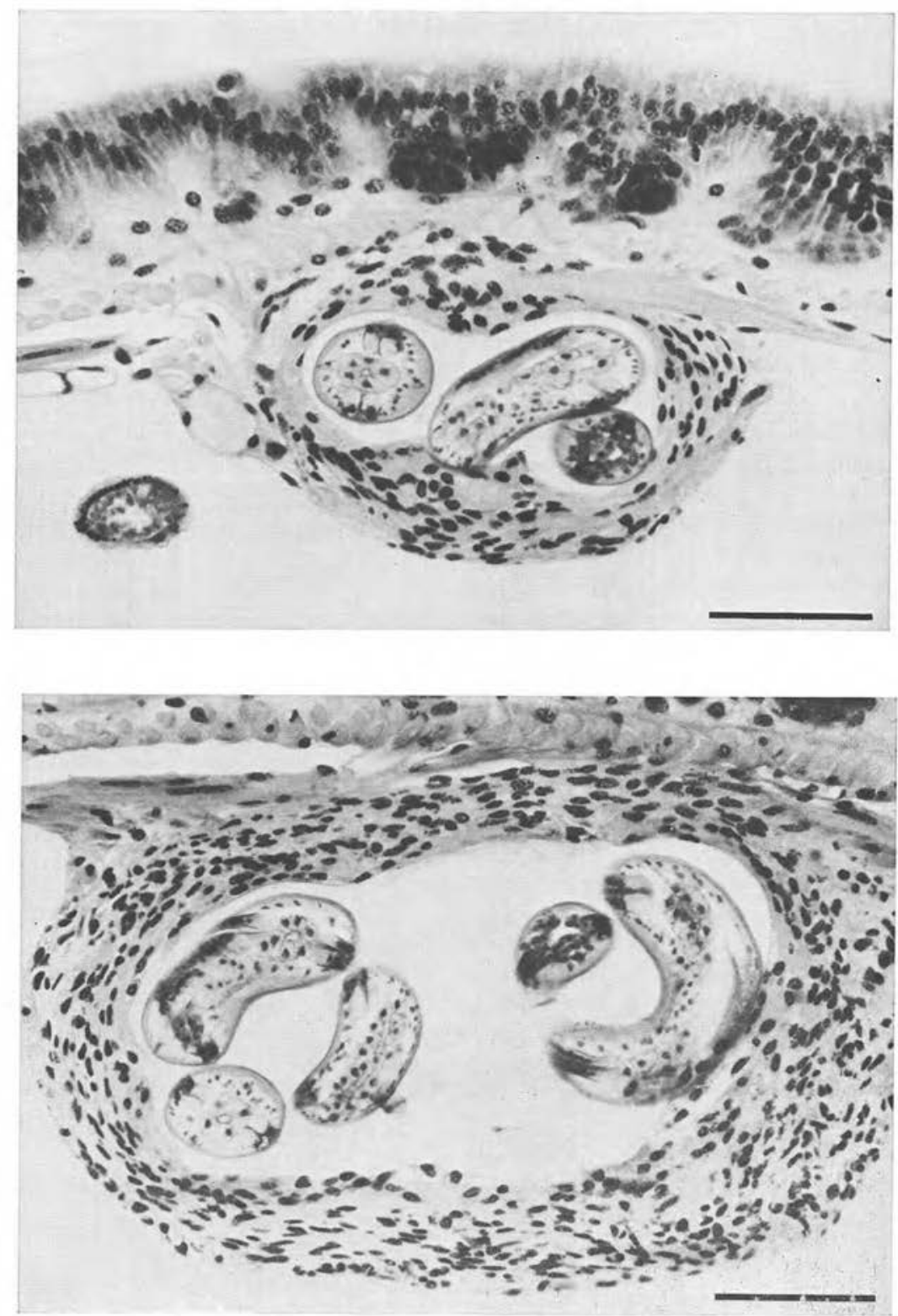

Fig. 7. Echinonema cinctum

En haut, 20 jours après l'infestation expérimentale du criquet, capsule hémocytaire contenant une larve. (Carnoy, hématoxyline de Groat, picro-indigo-carmin).

Pour cette figure, comme pour la suivante, le trait d'échelle reprásente $100 \mu \mathrm{m}$.

En bas, Capsule hémocytaire contenant deux larves. 
17 jours après l'infestation expérimentale, les capsules, qui ne contiennent en général qu'une seule larve, ont une forme de sphère aplatie de 200 à $300 \mu \mathrm{m}$ de diamètre (fig. 7, 1). Dans le cas de capsules contenant deux larves (fig. 7,2$)$, le diamètre peut atteindre 500 à $600 \mu \mathrm{m}$. La paroi des capsules a une épaisseur variant de 25 à $100 \mu \mathrm{m}$. Elle est constituée de plusieurs couches de cellules de petite taille caractéristiques d'un encapsulement hémocytaire (Seureau, 1973).

\section{- Discussion:}

La localisation, la taille et la structure des réactions cellulaires provoquées par les larves d'Echinonema cinctum chez le Criquet sont comparables à ce qui est observé après l'infestation expérimentale de cet Insecte par des Subulures (Seureau, 1973) et, dans la plupart des cas, par des Seuratidae (Quentin et Seureau, 1975). Cependant, et à la différence de l'encapsulement hétérogène des larves des Seuratidae, celui des larves d'Echinonema cinctum est uniquement hémocytaire, comme celui des larves des Subulures.

\section{V. - Conclusions}

La morphologie des Echinonematinae confirme entièrement les conclusions d'Inglis et de Quentin sur leur appartenance aux Seuratoidea-Seuratidae.

Si l'on considère la spinulation, appareil de fixation qui semble évoluer vers une complexité croissante, le genre Seuratum, parasite cosmopolite de Chiroptères (et accessoirement de Rongeurs archaïques), a une spinulation somatique simple. Le genre Seurechina, qui a une spinulation différenciée sur le cinquième antérieur du corps, est parasite d'un Dasyurinae : Dasyurus. Le genre Inglechina, qui acquiert les 3 rangs de gros crochets céphaliques, mais n'a pas de différenciation cervicale, est parasite d'un Dasyuridae Phascogalinae: Sminthopsis. Le genre Echinonema a non seulement les 3 rangs de gros crochets céphaliques, mais aussi une armature bien différenciée de la région cervicale. Il paraît donc être le genre le plus spécialisé, comme l'indique en outre sa monodelphie. Une espèce est parasite d'un Dasyuridae dans le Nord du continent et de nombreuses spéciations ont lieu, chez les Perameloidea, dans toute l'Australie.

Les Echinonematinae peuvent donc être considérés comme une branche issue d'ancêtres proches des Seuratum, parasites de Chiroptères, et adaptée à un groupe restreint de Marsupiaux australiens, les Dasyuroidea, puis les Perameloidea. Ceux-ci, selon Kirsch (1977), forment un ensemble qui se serait séparé des autres Marsupiaux vers le milieu du Paléocène. Contrairement aux données morphologiques, les données biologiques sur Echinonema indiquent des éléments plus primitifs que ceux du genre Seuratum. La larve, chez l'Insecte hôte intermédiaire, se développe à l'intérieur d'une capsule hémocytaire simple comme celle d'un Subuhuride. Cela entraîne, semble-t-il, une très faible croissance au cours du $2^{\mathrm{e}}$ stade larvaire, et la larve infestante ressemble donc plus à celle d'un Subuluride qu'à celle d'un Seuratide. 
REMERCIEMENTS. - Nous exprimons notre vive reconnaissance aux Docteurs Patricia Thomas-Mawson et David M. Spratt, qui ont bien voulu nous confier l'étude d'un abondant matériel et qui nous ont apporté d'importantes corrections en relisant le manuscrit.

\section{Bibliographie}

Inglis W. G.: The relationships of the Nematode Superfamily Seuratoidea. J. Helminthol., 1967, 41, 115-136.

Inglis W. G., Mawson P. M.: Miscellanea Nematodologica. VI. Echinonema australis sp. nov. from Sminthopsis crassicaudata in South Australia. J. Nat. Hist., 1967, 1, 173-175.

Johnston T. H., Mawson P.M. : Sundry Nematodes from Eastern Australian Marsupials. Trans. Roy. Soc. S.A., 1939, 63, 204-209.

Johnston T.H., Mawson P. M. : New and known Nematodes from Australian Marsupials. Proceed. Linnean Soc. New South Wales, 1940, 65, parts 5-6, 468-476.

Kirsch J.A.W.: The comparative serology of Marsupialia, and a classification of Marsupials. Aust. J. Zool., Suppl. Ser., 1977, No 52, 1-152.

Linstow O. von: Nemathelminthen. Von Herrn Richard Semon in Australien gesammelt. Denkschr. Med.-Naturw. Gesellsch. Jena, 1898, 8, 469-472.

Mackerras I. M., Mackerras M. J.: Taxonomy of the common short-nosed marsupial bandicoot of eastern Queensland. Australian J. Sci., 1960, 23, 51-52.

Quentin J.-C.: Cycle biologique de Subulura williaminglisi Quentin, 1965. Ontogenèse des structures céphaliques. Valeur phylogénétique de ce caractère dans la classification des Nématodes Subuluridae. Ann. Parasitol. Hum. Comp., 1969, 44, 451-484.

Quentin J.-C.: Sur le cycle évolutif de Seuratum cadarachense Desportes, 1947 et ses affinités avec ceux des Nématodes Subulures (Ascaridida) et Rictulaires (Spirurida). Ann. Parasitol. Hum. Comp., 1971, 45, 605-628.

Quentin J.-C., Seureau C. : Sur l'organogenèse de Seuratum cadarachense Desportes, 1947 (Nematoda, Seuratoidea) et les réactions cellulaires de l'Insecte Locusta migratoria, hôte intermédiaire. Z. Parasitenk., 1975, 47, 55-68.

Romer F.: Monotremata und Marsupialia. Zoologische Forschungsreisen in Australien und dem Malayischen Archipel. Fünfter Band: Systematik und Thiergeographie, I. Lieferung. Jenaische Denkschrif., 1894, 8, 151-160.

Seureau C. : Réactions cellulaires provoquées par les Nématodes Subulures et Spirurides chez Locusta migratoria (Orthoptère) : localisation et structure des capsules. Z. Parasitenk., 1973, 41, 119-138.

Yorke W., Maplestone P.A.: The Nematode parasites of Vertebrates. J. and A. Churchill., Publ., London, 1926, $536 \mathrm{p}$. 